\title{
In vitro inhibitory effect of two commercial probiotics on chromogenic actinomycetes
}

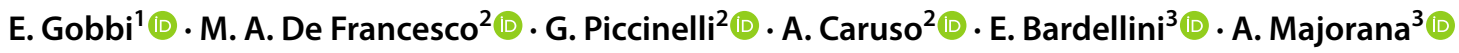

Received: 22 May 2019 / Accepted: 29 January 2020

(c) European Academy of Paediatric Dentistry 2020

\begin{abstract}
Purpose Black extrinsic discoloration is a common clinical and aesthetic problem. This study aims to evaluate the potential in vitro antagonistic activity of two commercial probiotics, Streptococcus salivarius M18 and Lactobacillus reuteri ProDentis, against microorganisms associated with black stains.

Methods Streptococcus salivarius M18 and Lactobacillus reuteri were tested against Aggregatibacter actinomycetemcomitans and Actinomyces naeslundiiusing their cell-free fermentative broth in a planktonic growth inhibition test.

Results Both probiotic cell-free supernatants showed the ability to reduce the pathogenic bacteria growth in a dose-dependent way. Streptococcus salivarius M18 showed a stronger antimicrobial activity than Lactobacillus reuteri ProDentis against the two indicator strains used. A. naeslundi was less susceptible to the probiotic activity of both $S$. salivarius and L. reuteri compared to A. actinomycetemcomitans.

Conclusions The obtained results demonstrate a potent antagonistic ability of probiotics to reduce the growth of microorganisms associated with black tooth stains. Therefore, these strains could be evaluated for a therapeutic use against dental pigmentations.
\end{abstract}

Keywords Aggregatibacter actinomycetemcomitans - Actinomyces naeslundii - Streptococcus salivarius M18 ·

Lactobacillus reuteri . Black stain

\section{Introduction}

Black extrinsic discoloration is a common clinical and aesthetic problem in childhood. Both primary and permanent teeth can be affected, with a reported prevalence of 1-20\% (Ronay and Attin 2011). Black stain (BS) is considered a special form of dental plaque unique for its insoluble iron salts and high calcium and phosphate contents (Hattab

E. Bardellini

elena.bardellini@unibs.it

1 Agrifood and Environmental Microbiology Platform (PiMiAA-AgroFood Lab), Department of Molecular and Translational Medicine, University of Brescia, Brescia, Italy

2 Department of Molecular and Translational Medicine, University of Brescia, Brescia, Italy

3 Department of Medical and Surgical Specialities, Radiological Sciences and Public Health, Dental School, Dental Clinic, University of Brescia, p.le Spedali Civili 1, 25133 Brescia, Italy et al. 1999; Reid and Beeley 1976). Previous culture-based studies have indicated an association between black tooth stain and chromogenic bacteria. While at first periodontal bacteria, such as Porphyromonas gingivalis, Prevotella intermedia and Prevotella melaninogenica were reported to be closely related to BS (Soukos et al. 2005), traditional and molecular examinations have proposed Actinomycetes as an etiological factor in the production of black pigment. Specifically, Aggregatibacter actinomycetemcomitans and Actinomyces naeslundii are reported as significantly predominant cultivable microorganisms found in patients with BSs (Saba et al. 2006; Li et al. 2015; Heinrich-Weltzien et al. 2014).

Current treatments focus on mechanical removal of the stains, which are hard to be removed by daily tooth brushing and are prone to resurface after professional scaling (Hattab et al. 1999). Therefore, further research on prevention and therapy of BSs is needed.

Probiotic bacteria are live microbial food supplements that confer a health benefit on humans (de Vrese and Schrezenmeir 2008). Their use to effect an improvement in 
oral health is a relatively undeveloped strategy and it has been investigated for prevention and treatment of dental caries, periodontal disease and for halitosis (Meurman 2005; Twetman and Stecksén-Blicks 2008; Allaker and Stephen 2017; Teughels et al. 2011).

To the best of our knowledge, no report on the introduction of beneficial bacterial population to help counter the proliferation of chromogenic species can be found in the published literature so far. Generally, probiotic activity is considered to influence the balance among the many species of commensal flora in the oral cavity. It is our view that probiotics may have beneficial applications in the reduction of BSs by inhibiting the proliferation of the BS associated bacteria, as demonstrated in vivo in a clinical trial (Bardellini et al. 2020), still the reasons why a probiotic intake inhibits the formation of BS requires further studies.

Among probiotics, Streptococcus salivarius possesses excellent credentials as an oral probiotic (Tagg and Dierksen 2003; Burton et al. 2011). It is a pioneer colonizer of the human oral cavity and produces bacteriocins, ribosomally synthesized proteinaceous antibiotics typically encoded by megaplasmid-borne loci (Wescombe et al. 2006). More specifically, the strain $S$. salivarius $\mathrm{M} 18$ has been shown to colonize and persist in the human oral cavity (Burton, Wescombe et al. 2013b), to reduce plaque formation (Burton and Drummond et al. 2013a) and to reduce gingivitis and periodontitis (Litty et al. 2015) Another probiotic is Lactobacillus reuteri, an obligate heterofermentative resident in the gastrointestinal tract in humans (Itsaranuwat et al. 2003) which is reported to produce antimicrobial substances with a broad-spectrum activity (Talarico et al. 1988; Ganzle et al. 2000), and it is effective against Streptococcus mutans (Caglar et al. 2008), as well as against periodontal pathogens (Vivekananda et al. 2010; Vicario et al. 2013).

To be able to display a probiotic effect against BSs a bacterium must compete with chromogenic bacteria, thus reducing the level of their colonization. The purpose of this study was to evaluate the inhibitory effects of two commercially available probiotics Streptococcus salivarius M18 and Lactobacillus reuteri Prodentis on growth inhibition of chromogenic bacteria, specifically $A$. actinomycetemcomitans and A. naeslundii, using their cellfree fermentative broth in a planktonic growth inhibition test.

\section{Materials and methods}

\section{Bacteria and bacterial culture}

Streptococcus salivarius M18 (IDA classification: DSM 14,865), also named by the manufacturer as BLIS M1 8
(BLIS Technologies, Dunedin, New Zealand), distributed as Carioblis ${ }^{\circledR}$ by Omeopiacenza (Pontenure, Italy), and Lactobacillus reuteri $\left(1 \times 10^{8} \mathrm{CFU}\right)$ strains DSM17938 and ATCC PTA5289 (Prodentis; BioGaia, Lund, Sweden) were used as probiotic strains. Two types of oral bacteria were used to test the antibacterial effect of these probiotic strains: Aggregatibacter actinomycetemcomitans DSM13386 (DSMZ, Braunschweig, Germany) and Actinomyces naeslundii DSM-11123 (DSMZ, Braunschweig, Germany). They were cultured using Schaedler agar with vitamin K and 5\% Sheep Blood (BD bioscience, Sparks, MD, USA) in anaerobic conditions.

\section{Antimicrobial activity of S. salivarius and L. reuteri against oral bacteria}

Bacterial strains were stored in tryptic soy broth (TSB) medium with $20 \%$ of glycerol at $-80{ }^{\circ} \mathrm{C}$ and used as required. Bacterial isolates (Streptococcus salivarius and Lactobacillus reuteri) from frozen stock were grown overnight and $500 \mu \mathrm{l}$ was then subcultured in brain heart infusion (BHI) medium (BD bioscience, Sparks, MD, USA) for $48 \mathrm{~h}$ at $37^{\circ} \mathrm{C}$ in anaerobic conditions. The bacterial suspensions were centrifuged at $4{ }^{\circ} \mathrm{C}$ for $10 \mathrm{~min}$ at $12.000 \mathrm{xg}$ and sterilized by filtration trough a $0.22 \mathrm{~mm}$ Millipore filter (Merck Millipore, Milano, Italy).

A test of the antibacterial properties of this medium was performed in accordance with recommendations from the Clinical and Laboratory Standards Institute (CLSI) (2012, 2016). A serial dilution was performed starting with $180 \mu 1$ of cell-free supernatants (CFS) of S. salivarius and L. reuteri in BHI medium to reach a proportion range of $10-90 \%$.

A. actinomycetemcomitans and $A$. naeslundii culture suspensions were diluted using fresh sterile BHI medium, so that the turbidity was equal to that of a McFarland 0.5 standard. Afterwards, $20 \mu \mathrm{l}$ of each bacterial suspension was inoculated in each well of a 96-well plate up to a final volume of $200 \mu \mathrm{l}$. BHI medium without inoculum and BHI medium with only the indicator bacteria were used as negative and positive control, respectively. The plate was then incubated for $24 \mathrm{~h}$ at $37{ }^{\circ} \mathrm{C}$ in an anaerobic chamber. Bacterial growth was measured at a wavelength of $600 \mathrm{~nm}$ using a microplate spectrometer. The entire experiment was performed three times with three independent repetitions.

\section{Statistical analysis}

The primary outcome of this study was the bacterial growth mean of A. actinomycetemcomitans and A. naeslundii culture suspensions evaluated when grown separately in BHI (controls) and in BHI added with different dilutions of CFS of S. salivarius or L. reuteri. Two hierarchical models were built, one for each culture, to estimate bacterial growth 
(measured 3 times per dilution, from 10 to $90 \%$ ) in relation to dilution (from 10 to 90\%) and "CASES" (control, CFS of $S$. salivarius, CFS of L. reuteri). Because normality assumptions were rejected, logarithm transformation was performed and estimates were than back-transformed to the original unit of measurement. Results are reported as estimates and $95 \%$ confidence intervals. All analyses were performed assuming a significance level of 5\% using the statistical software R (version 3.4.2).

\section{Results}

\section{Antibacterial activity of Streptococcus salivarius M18 and Lactobacillus reuteri}

The effect of the two probiotics on the chromogenic actinomycetes' growth was explored by adding increasing concentration of single CFS of the 48-h-old probiotic cultures to the culture medium of the actinomycetes.

Anti-A. actinomycetemcomitans activity of $S$. salivarius M18 occurred drastically at CFS concentrations of $40 \%$

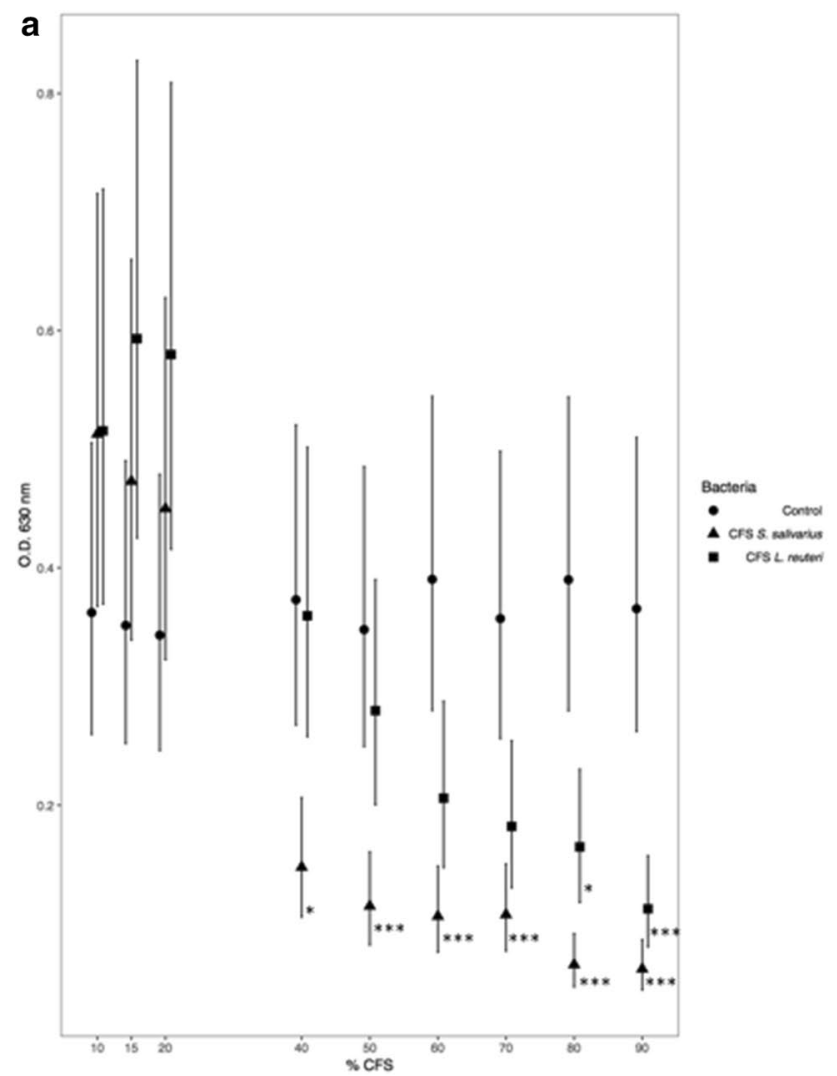

Fig. 1 Antibacterial activity of Streptococcus salivarius M18 and Lactobacillus reuteri ProDentis against Aggregatibacter actinomycetemcomitans (a) and Actinomyces naeslundii (b). CFS, cell-free supernatant. Each point represents the mean of three and higher (Fig. 1a) with a significant reduction of the $A$. actinomycetemcomitans' growth $(p$ value $=0.001)$ and a complete growth inhibition at $90 \%$. Differently, anti-A. actinomycetemcomitans activity of $L$. reuteri occurred at CFS concentrations higher than $60 \%(p$ value $=0.001)$ (Fig. 1a).

Anti-A. naeslundii activity of S. salivarius M18 occurred at concentrations higher than $40 \%$ with a complete growth inhibition at $90 \%$ (Fig. 1b), while anti-A. naeslundii activity of $L$. reuteri occurred at a concentration of $80 \%$ without reaching a complete growth inhibition at $90 \%$ (Fig. 1b). Both S. salivarius M18 and L. reuteri significantly reduced the $A$. naeslundii growth ranging between the concentrations of $40 \%$ ( $p$ value $<0.040$ ) and of $70 \%$ ( $p$ value $<0.0001$ ), respectively.

\section{Discussion}

BSs are characteristic pigmented deposits that may occur at any age but seem to peak in childhood with a decrease in prevalence during pubescence and adulthood. Even if not

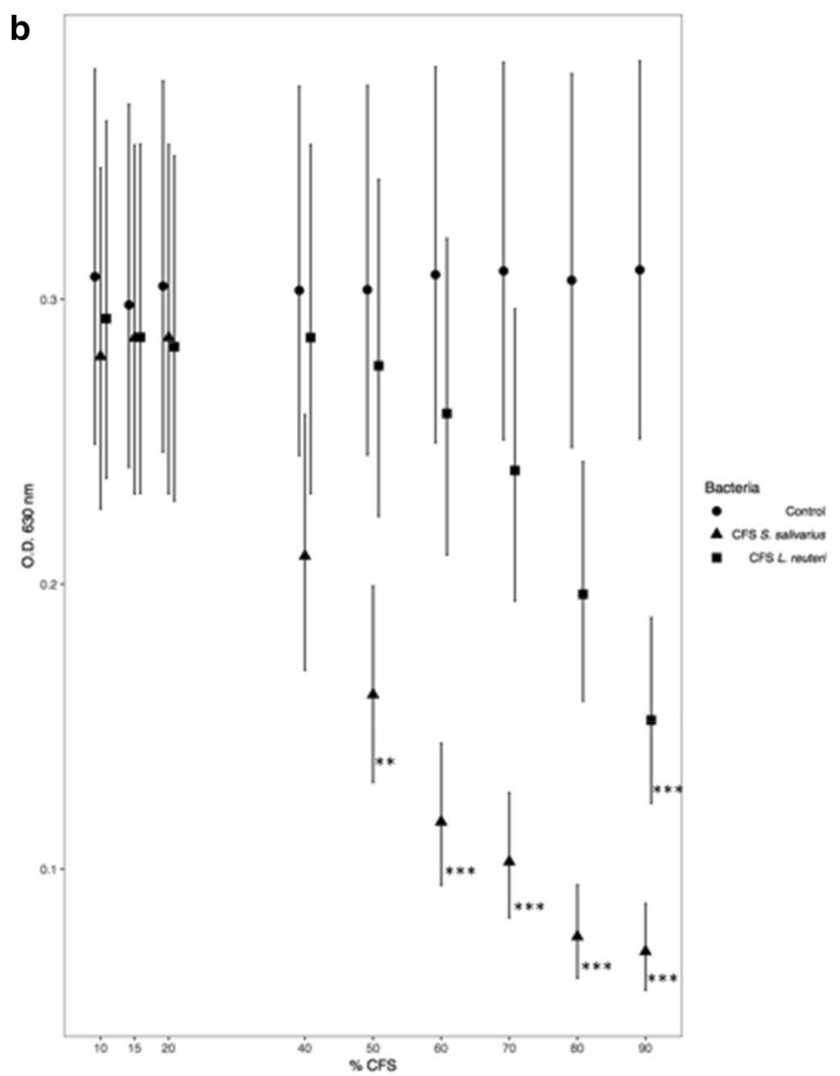

determinations ( $n=9$, average $+95 \%$ confidence interval). Asterisks are used to denote relevant comparisons and statistical significance as follows: $* p=0.001, * * p=0.0002, * * * p \leq 0.0001$ 
correlated with caries, BS represents an aesthetic problem, especially in the parents' eyes.

Probiotics are generally defined as microorganisms that confer a health benefit on humans by establishing a balance between beneficial and pathogenic bacteria (Koll et al. 2008; Li et al. 2015; Krasse et al. 2006). Their use is a relatively undeveloped strategy for prevention and treatment of caries and periodontitis (de Vrese and Schrezenmeir 2008).

BSs too are associated with a segregation of microbial communities in the oral cavity (Krasse et al. 2006), thus this approach has been considered as potentially beneficial in the prevention and treatment of BSs and applied for the first time in a clinical trial (Bardellini et al. 2020).

This short note is a preliminary contribution to unravel the underlying mechanisms. The study was undertaken to compare the in vitro efficiencies of two commercial probiotic products, including that used in the above mentioned clinical trial, against planktonic oral actinomycetes which are commonly reported as associated to BSs.

Both probiotics show antagonistic capacity, as their fermentative broth was able to significantly reduce the growth of the tested bacterial strains. Notably, in this study Streptococcus salivarius M18 showed a stronger antimicrobial activity than Lactobacillus reuteri ProDentis against the two indicator strains used. Furthermore, this study shows that $A$. naeslundi is less susceptible to the probiotic activity of both $S$. salivarius and $L$. reuteri compared to A. actinomycetemcomitans, whose growth is completely inhibited at high cell-free fermentative broth concentrations. This study also indicates a dosedependent inhibiting activity of the two probiotics as higher the concentrations of their fermentative broth used in the experiments, the higher was the reduction of the bacterial indicators growth.

The activities of $S$. salivarius and $L$. reuteri against these actinomycetes have already been reported, although strains and culture media may differ and generally the pathogenic bacteria were in association to periodontal diseases (Teughels et al. 2013; Kang et al. 2011; Twetman et al. 2009).

The nature of the antimicrobial activity exerted by the two probiotics in this work remains unclear. Because in this study, $\mathrm{pH}$ evaluation of CFS was not performed, the presented data do not support whether the inhibition is due to some secreted metabolite, present in the cell-free supernatants of $S$. salivarius and $L$. reuteri or due to organic acids production, which could contribute to reducing the number of indicator bacteria. However, some previous studies demonstrated that the acidic environment provided by Lactobacillus strains can directly affect their antibacterial activities against $S$. mutans (Lin et al. 2015; Keller et al. 2011). Therefore, the role of the acids produced by probiotic strains on bacterial growth is still unclear.
This is corroborated by many reports showing that Lactobacilli can produce organic acids, $\mathrm{H}_{2} \mathrm{O}_{2}$ and bacteriocins (Cintas et al. 2001) while $S$. salivarius M18 releases into saliva copious quantities of bacteriocins and enzymes as dextranase and urease (Heng et al. 2011). Additional research is required to verify the mechanism of antibacterial activity found here and if and which probiotic metabolites are eventually produced in vitro and in vivo during the interactions studied in this work and what is their role in the oral cavity for preventing BSs.

This study ascertained in vitro the potential antagonistic activity of two commercial products, Streptococcus salivarius M18 and Lactobacillus reuteri ProDentis: these strains are to be considered for a therapeutic use against dental pigmentations.

Acknowledgements The Authors are grateful to Dr Veronica Cappa for the statistical support.

\section{Compliance with ethical standards}

Conflict of interest The Authors declare that they have no conflict of interest.

Ethical approval This study did not involve any human participants or animals.

Informed consent This study did not need informed consent from the patients, being an in vitro study.

\section{References}

Allaker RP, Stephen AS. Use of probiotics and oral health. Curr Oral Health Rep. 2017;4:309-18.

Bardellini E, Amadori A, Gobbi E, et al. Does Streptococcus Salivarius strain M18 assumption make black stains disappear in children? Oral Health Prev Dent. 2020 (in press).

Burton JP, Cowley S, Simon RR, et al. Evaluation of safety and human tolerance of the oral probiotic Streptococcus salivarius K12: a randomized, placebo-controlled, double-blind study. Food Chem Toxicol. 2011;49:2356-64.

Burton JP, Drummond BK, Chilcott CN, et al. Influence of the probiotic Streptococcus salivarius strain M18 on indices of dental health in children: a randomized double-blind, placebo-controlled trial. J Med Microbiol. 2013a;62:875-84.

Burton JP, Wescombe PA, Macklaim JM, et al. Persistence of the oral probiotic Streptococcus salivarius M18 is dose dependent and megaplasmid transfer can augment their bacteriocin production and adhesion characteristics. PLoS ONE One. 2013b;8:e65991.

Caglar E, Kuscu OO, Cildir SK, et al. A probiotic lozenge administered medical device and its effect on salivary mutans streptococci and lactobacilli. Int J Paediatr Dent. 2008;18:35-9.

Cintas LM, Casaus MP, Herranz C, et al. Bacteriocins of lactic acid bacteria. Food Sci Tech Int. 2001;7:281-305.

Clinical and Laboratory Standards Institute. Methods for antimicrobial susceptibility testing of anaerobic bacteria. Approved standardEight Edition, CLSI document M11-A8. Wayne: Clinical and Laboratory Standards Institute; 2012. 
Clinical and Laboratory Standards Institute. Methods for dilution antimicrobial susceptibility tests for bacteria that grow aerobically, M7-A10. Wayne: Clinical and Laboratory Standards Institute; 2016.

de Vrese M, Schrezenmeir J. Probiotics, prebiotics, and synbiotics. Adv Biochem Eng Biotechnol. 2008;111:1-66.

Ganzle MG, Holtzel A, Walter J, et al. Characterization of reutericyclin produced by Lactobacillus reuteri LTH2584. Appl Environ Microbiol. 2000;66:4325-33.

Hattab FN, Qudeimat MA, AlRimawi HS. Dental discoloration: an overview. J Esthet Restor Dent. 1999;11:291-310.

HeinrichWeltzien R, Bartsch B, Eick S. Dental caries and microbiota in children with black stain and non-discoloured dental plaque. Caries Res. 2014;48:118-25.

Heng NCK, HajiIshak NS, Kalyan A, et al. Genome sequence of the bacteriocin-producing oral probiotic Streptococcus salivarius Strain M18. J Bacteriol. 2011;193:6402-3.

Itsaranuwat P, Al-Haddad KSH, Robinson RK. The potential therapeutic benefits of consuming 'health promoting' fermented dairy products: a brief update. Int J Dairy Technol. 2003;56:203-10.

Kang MS, Oh JS, Lee HC, et al. Inhibitory effect of Lactobacillus reuteri on periodontopathic and cariogenic bacteria. J Microbiol. 2011;49:193-9.

Keller MK, Hasslof P, Stecksen-Blicks C, et al. Co-aggregation and growth inhibition of probiotic lactobacilli and clinical isolates of mutans streptococci; an in vitro study. Acta Odontol Scand. 2011;69:263-8.

Koll P, Mandar R, Marcotte H, et al. Characterization of oral lactobacilli as potential probiotics for oral health. Oral Microbiol Immunol. 2008;23:139-47.

Krasse P, Carlsson B, Dahl C, et al. Decreased gum bleeding and reduced gingivitis by the probiotic Lactobacillusreuteri. Swed Dent J. 2006;30:55-60.

Li Y, Zhang Q, Zhang F, et al. Analysis of the microbiota of black stain in the primary dentition. PLoS ONE One. 2015;10:e0137030. https://doi.org/10.1371/journal.pone.0137030.

Lin X, Chen X, Chen Y, et al. The effect of five probiotic lactobacilli strains on the growth and biofilm formation of Streptococcus mutans. Oral Dis. 2015;21:e128-e134134.

Litty S, Nagarathna D, Merline V. Probiotics in periodontal therapy. Int J Pharm Bio Sci. 2015;6:242-50.

Meurman JH. Probiotics: do they have a role in oral medicine and dentistry? Eur J Oral Sci. 2005;113(3):188-96.

Reid JS, Beeley JA. Biochemical studies on the composition of gingival debris from children with black extrinsic tooth stain. Caries Res. 1976;10:363-9.
Ronay V, Attin T. Black stain-a review. Oral Health Prev Dent. 2011;9:37-45.

Saba C, Solidani M, Berlutti F, et al. Black stains in the mixed dentition: a PCR microbiological study of the etiopathogenic bacteria. J Clin Pediatr Dent. 2006;30:219-24.

Soukos NS, Som S, Abernethy AD, et al. Phototargeting oral black-pigmented bacteria. Antimicrob Agents Chemother. 2005;49:1391-6.

Tagg JR, Dierksen KP. Bacterial replacement therapy: adapting 'germ warfare' to infection prevention. Trends Biotechnol. 2003;21:217-23.

Talarico TL, Casas IA, Chung TC, et al. Production and isolation of reuterin, a growth inhibitor produced by Lactobacillus reuteri. Antimicrob Agents Chemother. 1988;32:1854-8.

Teughels W, Loozen G, Quirynen M. Do probiotics offer opportunities to manipulate the periodontal oral microbiota? J Clin Periodontol. 2011:38:159-77.

Teughels W, Durukan A, Ozcelik O, et al. Clinical and microbiological effects of Lactobacillus reuteri probiotics in the treatment of chronic periodontitis: a randomized placebo-controlled study. J Clin Periodontol. 2013;40:1025-35.

Twetman S, Stecksén-Blicks C. Probiotics and oral health effects in children. Int J Paediatr Dent. 2008;18:3-10.

Twetman S, Derawi B, Keller M, et al. Short-term effect of chewing gums containing probiotic Lactobacillus reuteri on the levels of inflammatory mediators in gingival crevicular fluid. Acta Odontol Scand. 2009;67:19-24.

Vicario M, Santos A, Violant D, et al. Clinical changes in periodontal subjects with the probiotic Lactobacillus reuteri Prodentis: a preliminary randomized clinical trial. Acta Odontol Scand. 2013;71:813-9.

Vivekananda MR, Vandana KL, Bhat KG. Effect of the probiotic Lactobacilli reuteri (Prodentis) in the management of periodontal disease: a preliminary randomized clinical trial. J Oral Microbiol. 2010;2:5344.

Wescombe PA, Burton JP, Cadieux PA, et al. Megaplasmids encode differing combinations of antibiotics in Streptococcus salivarius. Antonie Van Leeuwenhoek Van Leeuwenhoek. 2006;90:269-80.

Publisher's Note Springer Nature remains neutral with regard to jurisdictional claims in published maps and institutional affiliations. 